\title{
Does experience play a role in the ability to perform liver stiffness measurements by means of supersonic shear imaging (SSI)?
}

\author{
Oana Grădinaru-Taşcău, Ioan Sporea, Simona Bota, Ana Jurchiş, Alina Popescu, \\ Mădălina Popescu, Roxana Şirli, Milana Szilaski
}

Department of Gastroenterology and Hepatology,"Victor Babeş" University of Medicine and Pharmacy, Timişoara, Romania

\begin{abstract}
The aim of this study was to identify if the experience of the examiner does plays a role in the ability to perform liver stiffness (LS) measurements by means of supersonic shear imaging (SSI) due to the lack of recommendation regarding this issue. Material and methods: The study included 371 consecutive subjects ( $42 \%$ men and 58\% women, with a median age of 48 years, ranging between 17-85 years) with or without hepatopathies, in which LS was evaluated with an AixplorerTM ultrasound system (SuperSonic Imagine S.A., Aix-en-Provence, France). Reliable LS measurements by means of SSI were defined as the median value of 5 LS measurements expressed in kilopascals $(\mathrm{kPa})$. The SSI measurements were performed by a novice (with less than 300 abdominal ultrasound examinations performed) or by a more experienced operator (with approximately 500 ultrasound examinations performed). The results of both operators were compared. Results: The novice performed $57.4 \%$ and the more experienced operator $42.6 \%$ of the SSI measurements. The more experienced operator had a higher rate of reliable examinations as compared with the novice: $87.4 \%$ vs. $72.8 \%(\mathrm{p}=0.001)$. The rate of reliable measurements was similar for novice and experienced operator in patients with a normal weight (BMI $<25 \mathrm{~kg} / \mathrm{m} 2)$ and in overweight patients (BMI between $25.1-29.9 \mathrm{~kg} / \mathrm{m} 2), 92.3 \%$ vs. $97.5 \%$, p=0.24, respectively $71.1 \%$ vs $80.4 \%, \mathrm{p}=0.39$. For obese patients $(\mathrm{BMI} \geq 30 \mathrm{~kg} / \mathrm{m} 2)$ the rate of reliable LS measurements was significantly higher for the more experienced operator as compared with the novice: $73.4 \%$ vs $45.9 \%, p=0.03$. Conclusions: It seems that experience in liver ultrasonography plays a role in the ability to perform LS measurements by means of SSI and leads to achieving more reliable LS measurements especially in obese subjects.

Keywords: liver stiffness, Supersonic Shear Imaging, Transient Elastography, Acoustic Radiation Force Impulse elastography
\end{abstract}

\section{Introduction}

Chronic liver disease still represents a frequent pathology among people of all ages, regardless of its etiology (viral chronic hepatitis B, C or non-viral hepatopathies). Even if the scientific area of research in this domain is vast, until now, a "perfect" method for assessing the severity of the liver disease has not been developed. For decades the "gold standard" method for the evaluation of the chronic liver diseases' severity has been considered to be the liver biopsy (LB) [1].This invasive maneuver

Received 30.05.2013 Accepted 25.06.2013

Med Ultrason

2013, Vol. 15, No 3, 180-183

Corresponding author: Oana Grădinaru-Taşcău

10 Iosif Bulbuca Str.

300736 Timişoara, Romania

Tel: +40727341527, Fax: +40256488003

Email: bluonmyown@yahoo.com has its limitations due to complications that can appear during and after the procedure [2], to the small size of the harvested specimen, and also due to the irregular disposition of fibrosis in the liver [3]. For several years there was an increasing interest in the non-invasive methods for assessing the severity of the liver disease, serological tests being the first ones to be developed followed by elastographic methods such as strain elastography: Real-time Tissue Elastography-Hi-RTE [4], and shear wave elastography: Acoustic Radiation Force Impulse (ARFI) [5] or SuperSonic Shear Imaging (SSI) [6]. The most studied shear wave elastographic method is Transient Elastography (TE) - Fibroscan (Echosens, France), proven to be a reliable tool for the assessment of liver fibrosis severity by several published studies and meta-analyses. Also, a meta-analysis [7] regarding the performance o ARFI for liver fibrosis assessment has been published, showing favorable results. 
SSI is the newest shear wave elastographic method [6]. The advantages of this new technique are: the use of a ultrafast, ultrasonic scanner; the system is included in a standard ultrasound system; patients with ascites can also be evaluated; the final result is presented in both color coded image and numeric value; the resulting value can be converted in units of shear wave velocity $(\mathrm{m} / \mathrm{s})$ or units of Young's modulus (kiloPascals); but very few studies have been published until now regarding this elastographic method.

The aim of this study was to identify if experience plays a role in the ability to perform fibrosis assessment by means of SSI because until now there are no recommendations regarding this issue and it is a known fact that ultrasonography is an operator dependent technique.

\section{Material and methods}

The study group included 371 consecutive subjects: healthy volunteers (without a medical background of liver disease, with a normal abdominal ultrasound, without any additional testing), patients with chronic hepatitis B or C, patients with non-viral hepatitis (alcoholic or nonalcoholic steatohepatitis), and patients diagnosed with liver cirrhosis.

All subjects were asked for their consent before any investigational procedure was performed in accordance with the Helsinki Declaration of 1975 and the study was approved by the local Ethics Committee.

In each patient the liver stiffness was evaluated with an Aixplorer ${ }^{\mathrm{TM}}$ ultrasound system (SuperSonic Imagine S.A., Aix-en-Provence, France), with a SC 6-1 broadband convex probe. Measurements were performed in supine position, with the right arm in maximum abduction, between the ribs, in the right liver lobe, $2 \mathrm{~cm}$ under the liver capsule, in the best acquired acoustic window for liver evaluation, in an area of parenchyma free of large vessels, in normal breathing, by two operators, a novice (with less than 300 abdominal ultrasound examinations performed) or by a more experienced operator (with approximately 500 ultrasound examinations performed) (fig 1). Reliable LS measurements by means of SSI were defined as the median value of 5 LS measurements expressed in kilopascals $(\mathrm{kPa})$.

\section{Statistical analysis}

The statistical analysis was performed using the MedCalc Software (MedCalc program, Belgium). For numerical variables with non-normal distribution, median values and range intervals were used. Qualitative variables were presented as numbers and percentages. Non-

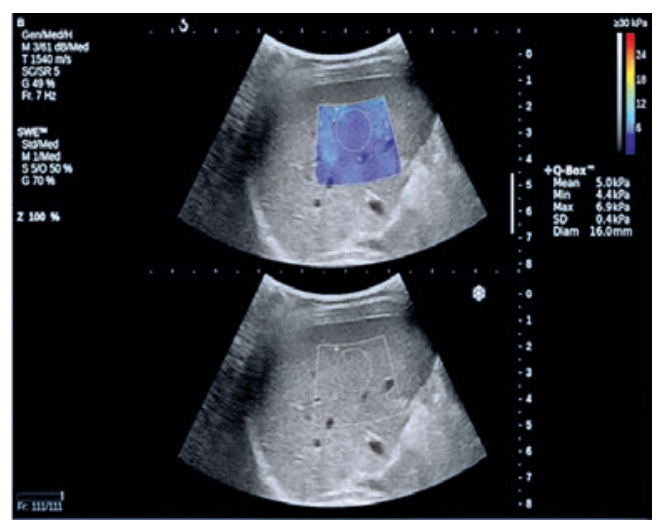

Fig 1. An elastographic image of mild fibrosis on the Aixplorer system

parametric tests were used for variables with non-normal distribution and parametric tests (t-test) were used for the assessment of differences between variables with normal distribution. A $p$-value $<0.05$ was considered significant for the statistic test.

\section{Results}

The study group included 371 consecutive subjects, $42 \%$ men and $58 \%$ women, with a median age of 48 years (ranging between 17-85 years) and a median body mass index (BMI) of $26.5 \mathrm{~kg} / \mathrm{m}^{2}$ (ranging between 18 $\left.35 \mathrm{~kg} / \mathrm{m}^{2}\right)$.

The novice performed $57.4 \%$ and the more experienced operator $42.6 \%$ of the SSI measurements. The more experienced operator had a significantly higher rate of reliable examinations compared with the novice: $87.4 \%$ vs. $72.8 \%(\mathrm{p}=0.001)$.

The rate of reliable measurements was similar for the novice and the experienced operator in patients with a normal weight (BMI $\left.<25 \mathrm{~kg} / \mathrm{m}^{2}\right)$ and in overweight patients (BMI between $25.1-29.9 \mathrm{~kg} / \mathrm{m}^{2}$ ), $92.3 \%$ vs. $97.5 \%, \mathrm{p}=0.24$, respectively $71.1 \%$ vs $80.4 \%, \mathrm{p}=0.39$.

In obese patients $\left(B M I \geq 30 \mathrm{~kg} / \mathrm{m}^{2}\right)$ the rate of reliable LS measurements was significantly higher for the more experienced operator as compared with the novice: $73.4 \%$ vs $45.9 \%, \mathrm{p}=0.03$.

\section{Discussions}

The burden of chronic liver disease is still high due to the high prevalence of the $\mathrm{B}$ and $\mathrm{C}$ viral hepatitis along with the alcoholic and non-alcoholic fatty liver disease. 
To manage this expanding pool of patients, accurate, noninvasive tools for staging liver fibrosis are needed.

Before the non-invasive methods were developed, the most frequently used method for liver fibrosis evaluation was the abdominal ultrasound; a real time technique, affordable, non irradiating, well tolerated, but an operator dependent method, in which the practical experience is needed for a good quality ultrasound examination [8]. Based on these facts, this study tried to assess if previous experience in abdominal ultrasound influences the ability to obtain reliable results by SSI - an ultrasound based elastographic method.

The non-invasive elastographic methods for liver fibrosis evaluation that were studied the most are strain elastography-HI-RTE [4], and share wave elastographic methods: TE [9] and ARFI [5]. Several studies evaluated the influence of the operator in each of these methods.

In a study by Boursier et al [10], ARFI measurements were performed in 101 patients by two operators: an expert and a novice and their conclusion was that interobserver agreement was very good $(\mathrm{Ric}=0.84)$ and that ARFI is a feasible and reproducible technique. Regarding the reproducibility of interobserver in ARFI, Bota et al [11] showed a statistically significant correlation. In the same study, Bota et al [11] stated that technical parameters such as interquartile range (IQR) and success rate improve the accuracy of ARFI measurements in predicting liver fibrosis assessment and recommend using them in clinical practice. Another study by Popescu et al [12] recommended that ARFI measurements should be performed in fasting conditions, as it was shown that ARFI measurements' values are higher after food intake as compared to those obtained in fasting conditions.

In the study of Gheonea et al [13] regarding the interobserver variability when performing elasticity measurements by means of Hitachi sono-elastography, 97 patients were evaluated by two physicians: a beginner and an experienced ultrasonographist, the statistical results showed that the more experienced physician obtained higher quality and more accurate recordings.

Regarding the role of experience in Transient Elastography, Castera et al [14] reported that operators who had performed fewer than $500 \mathrm{TE}$ examinations had a 2.5 -fold increased odds of obtaining unreliable LS measurements results compared with more experienced operators.

In a study by Hudson et al [15] regarding the reliability of shear wave elastography in SSI on 15 patients, the intra-operator reproducibility of measurements performed on the same day was better than the inter-operator reproducibility, with no significant differences between subjects with a BMI less or greater than $25 \mathrm{~kg} / \mathrm{m}^{2}$.
The present study showed that experience in ultrasound is important for liver fibrosis assessment by means of SSI, when patients are obese, with a poor acoustic window, thus more difficult to examine by ultrasound. Also the results of this study showed that previous experience in liver ultrasound is a factor associated with a higher percentage of valid measurements in obese patients.

To our knowledge, our study is the largest to assess if previous experience in abdominal ultrasonography makes a difference in LS measurements by means of SSI. Ferraioli et al [16] evaluated SSI reproducibility on 42 subjects, in this study the expert operator having a higher reproducibility than the novice operator.

In conclusion, it seems that experience in liver ultrasonography plays a role in the ability to perform LS measurements by means of SSI and leads to achieving more reliable LS measurements especially in obese subjects.

\section{Conflict of interest: none}

\section{References}

1. Rockey DC, Caldwell SH, Goodman ZD, Nelson RC, Smith $\mathrm{AD}$; American Association for the Study of Liver Diseases. Liver biopsy. Hepatology 2009; 49: 1017-1044.

2. Piccinino F, Sagnelli E, Pasquale G, Giusti G. Complications following percutaneous liver biopsy. A multicentre retrospective study on 68,276 biopsies. J Hepatol 1986; 2: $165-173$.

3. Bedossa P, Dargere D, Paradis V. Sampling variability of liver fibrosis in chronic hepatitis C. Hepatology 2003; 38: 1449-1457.

4. Havre RF, Elde E, Gilja OH, et al. Freehand real-time elastography: impact of scanning parameters on image quality and in vitro intra-and interobserver validations. Ultrasound Med Biol 2008; 34: 1638-1650.

5. Friedrich-Rust M, Ong MF, Herrmann E, et al. Real-time elastography for noninvasive assessment of liver fibrosis in chronic viral hepatitis. AJR Am J Roentgenol 2007; 188: 758-764.

6. Bavu E, Gennisson JL, Couade M, et al. Noninvasive in vivo liver fibrosis evaluation using supersonic shear imaging: a clinical study on 113 hepatitis $C$ virus patients. Ultrasound Med Biol 2011; 37: 1361-1373.

7. Friedrich-Rust M, Nierhoff J, Lupsor M, et al. Performance of Acoustic Radiation Force Impulseimaging for the staging of liver fibrosis: a pooled meta-analysis. J Viral Hepat 2012; 19: e212-e219.

8. Minimum Training Requirements for the Practice of Medical Ultrasound in Europe. EFSUMB Educational \& Professional Standards Committee, January 2003.

9. Friedrich-Rust M, Ong MF, Martens S, et al. Performance of transient elastography for the staging of liver fibrosis: a meta-analysis. Gastroenterology 2008; 134: 960-974. 
10. Boursier J, Isselin G, Fouchard-Hubert I, et al. Acoustic radiation force impulse: a new ultrasonographic technology for the widespread noninvasive diagnosis of liver fibrosis Eur J Gastroenterol Hepatol 2010; 22: 1074 1084.

11. Bota S, Sporea I, Şirli R, Popescu A, Dănilă M, Șendroiu $M$. Factors that influence the correlation of acoustic radiation force impulse (ARFI), elastography with liver fibrosis. Med Ultrason 2011; 13: 135-140.

12. Popescu A, Bota S, Sporea I, The influence of food intake on liver stiffness values assessed by acoustic radiation force impulse elastography-preliminary results. Ultrasound Med Biol 2013; 39: 579-584.
13. Gheonea DI, Săftoiu A, Ciurea T, et al. Real-time sonoelastography in the diagnosis of diffuse liver diseases. World J Gastroenterol 2010; 16: 1720-1726.

14. Castéra L, Foucher J, Bernard PH, et al. Pitfalls of liver stiffness measurement: a 5-year prospective study of 13,369 examinations. Hepatology 2010; 51: 828-835.

15. Hudson JM, Milot L, Parry C, Williams R, Burns PN. Inter- and intra-operator reliability and repeatability of shear wave elastography in the liver: a study in healthy volunteers. Ultrasound Med Biol 2013; 39: 950-955.

16. Ferraioli G, Tinelli C, Zicchetti M, et al. Reproducibility of real-time shear wave elastography in the evaluation of liver elasticity. Eur J Radiol 2012; 81: 3102-3106. 